CASE REPORT

\title{
Adult Onset Still's Disease: A Case Report
}

\author{
*MR Siddiqui ${ }^{1}$, GK Khan ${ }^{2}$, MS Islam³ ${ }^{3}$ KMA Ahmed ${ }^{4}$ \\ $1 *$ Dr. Mahmudur Rahman Siddiqui, Assistant Professor, Department of Medicine, Anwer Khan Modern \\ Medical College \& Hospital, Dhaka \\ ${ }^{2}$ Prof. (Dr.) Golam Kibria Khan, Professor of Medicine, Department of Medicine, Dhaka Medical \\ College Hospital, Dhaka \\ ${ }^{3}$ Dr. Md. Safiqul Islam, Postgraduate resident, Department of Medicine, Dhaka Medical College \\ Hospital, Dhaka \\ ${ }^{4}$ Dr. K. M. Ahasan Ahmed, Postgraduate resident, Department of Medicine, Dhaka Medical College \\ Hospital, Dhaka \\ *Corresponding Author
}

\begin{abstract}
Adult onset Still's disease (AOSD) is a chronic multi-system inflammatory disorder characterized by high spiking fever, polyarthralgia and skin rash. Lymphadenopathy is another prominent feature of adult onset Still's disease. We describe a 22 years old lady presented with fever, skin rashes and polyarthritis for 3 months. Examination revealed fever, typical skin rash, generalized lymphadenopathy and polyarthritis. On investigation there were neutrophilic leukocytosis, high ESR, high ferritin level, but RA test and ANA test were negative. All of her history, clinical examinations and laboratory findings fulfill the diagnostic Yamaguchi criteria for AOSD. With proper treatment, now she is completely symptoms free and leaving a healthy life.
\end{abstract}

Key words: Fever, skin rash, polyarthritis, Adult onset Still's disease

\section{Introduction}

Adult onset Still's disease (AOSD) is a chronic systemic inflammatory disorder of unknown etiology, and its major clinical manifestations include high spiking fever, sore throat, muscle pain, polyarthralgia, salmon colored evanescent rash, hepatosplenomegaly, lymphadenopathy and neutrophilic leukocytosis ${ }^{1,2}$. AOSD is rare and has a bimodal age distribution in all ethnic groups with peaks at 15-25 and 36-46 years of age in both sexes with an incidence of 0.16 cases $/ 100000$ persons/year ${ }^{1,2}$. In fact there is no cure for adult Still's disease; however, treatment may offer symptom relief for adult Still's disease and help to prevent complication. As it progresses, adult Still's disease may lead to chronic arthritis and other complications. Recently we have diagnosed a case of AOSD in our hospital, who became complete symptoms free with proper treatment, though it is non curable.

\section{Case Report}

22 years of age normotensive, nondiabetic women admitted at department of Medicine, Dhaka Medical College Hospital with the complaints of fever, multiple joint pain and skin rashes over whole body for last 3 months. Fever was high grade continuous in nature. Generalized macular rashes especially in upper limbs, chest and back, with symmetrical polyarthritis involving wrist, proximal interphalangeal joints (PIP), metacarpophalangeal joints (MCP), knees and ankles. On quarry she also gave no history of sore throat for last 1 month. There were no early morning stiffness, ocular symptoms, orogenital ulcers, urinary symptoms, photophobia, and contact to infected person or major systemic symptoms. 
Examination revealed well built, oriented young lady with macular rashes over upper limbs, chest and lower back and there was no rash over his finger knuckles. The patient was febrile $103^{\circ} \mathrm{F}$ with regular heart rate $90 \mathrm{~b} / \mathrm{min}$, blood pressure of $110 / 70 \mathrm{mmHg}$ and normal jugular venous pressure, but there were generalized lymphadenopathy involving bilateral anterior, posterior cervical groups and inguinal groups. She had acute synovitis of ankles, wrists and PIP joints, mainly of ring fingers with weak hand grip bilaterally, but full range of movements of all locomotor system and no proximal or distal muscular weakness. Deep tendon reflexes were normal. Examination of chest, abdomen, central and peripheral nervous systems were unremarkable.

Investigations revealed haemoglobin $7.5 \mathrm{gm} / \mathrm{dl}$, erythrocyte sedimentation rate (ESR) $135 \mathrm{~mm}$ in 1st hour, total white blood cell count $12.17 \mathrm{x}$ $10^{9} / \mathrm{L}$, neutrophil $67 \%$, lymphocyte $14 \%$, peripheral blood film suggestive of anaemia of chronic disorder with neutrophilic leukocytosis and marked roulex formation. Urine routine examination was normal, no growth in urine culture. Liver function test was normal, RA test negative, ANA - negative, ultrasonography of the whole abdomen was normal, but there was very high ferritin - $4011 \mathrm{ng} / \mathrm{ml}$ (normal 15-120ng/ml).

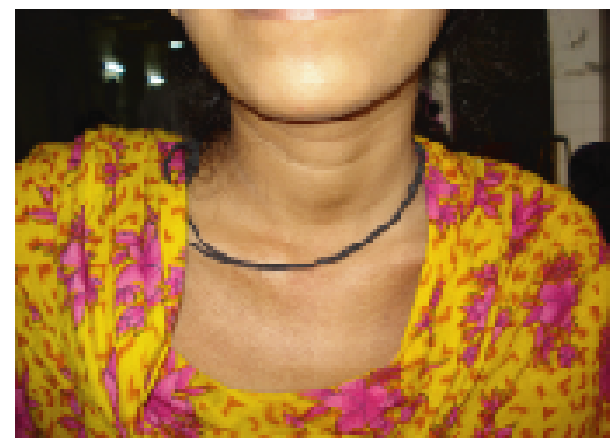

Fig 1: Patient of AOSD with skin rashes

Finally AOSD diagnosis was made according to Yamaguachi criteria (she is having 4 major features and 3 minor features) and she was started on prednisolone 40mg daily and NSAID (Diclofenac sodium $100 \mathrm{mg}$ daily) after which she became afebrile for the first time in last 2 months after onset of illness. The patient showed considerable improvement and was discharged home after one week on prednisolone 40mg daily with tapering dose of $5 \mathrm{mg}$ weekly, Diclofenac sodium and omeprazol $20 \mathrm{mg}$ twice daily. With proper treatment, now she is completely symptoms free and leaving a healthy life.

\section{Discussion}

Still's disease is named after an English doctor named George Still, who described the condition in children in 1897. George Still published his monograph, "On a Form of Chronic Joint Disease in Children." 3 Still's disease is now known as systemic onset juvenile rheumatoid arthritis (JRA). Bywaters in 1971, the term "adult Still's disease" was used to describe adults who had a condition similar to systemic onset $\mathrm{JRA}^{2}$. Although cause of AOSD is unknown, the condition may be triggered by a viral or bacterial infection.

High-sensitivity classification criteria have been proposed, since there is no single test to establish the diagnosis (Table 1$)^{2}$.

Table I: Classification criteria for adult-onset Still's disease proposed by Yamaguchi et $\mathrm{al}^{2}$

\begin{tabular}{ll}
\hline \multicolumn{1}{c}{ Major criteria } & \multicolumn{1}{c}{ Minor criteria } \\
\hline - Temperature of $>39^{0} \mathrm{C}$ for $>1 \mathrm{wk}$. & - Sore throat \\
- Leukocytosis $>10000 / \mathrm{cu} \mathrm{mm}$ and & - Lymph node enlargement \\
- including $80 \%$ more of granulocytes. & - Splenomegaly \\
- Typical rash. & - Liver dysfunction (high ALT) \\
- Arthralgias $>2$ wk. & - Negative ANA, RF \\
\hline
\end{tabular}

After excluding infections, malignancies, and other rheumatic diseases, adult Still's should be considered if 5 criteria ( 2 of which being major ones) are present. ANA = antinuclear antibody; $\mathrm{RF}=$ rheumatoid factor. (Yamaguchi criteria 1992: specificity $92 \%$ and sensitivity $96 \%$ )

A salmon-pink bumpy or flat rash (87\% cases) may come and go with the fever. The rash usually appears on trunk, arms or legs 4,5 . Physical contact such as rubbing of skin may provoke the rash to appear. In $90 \%$ cases symmetrical or asymmetrical polyarthralgia or arthritis present, which usually involves knees, wrists, ankles, elbows, hands, shoulders joints and cervical spines ${ }^{4,5}$. Usually, the joint discomfort 
lasts at least two weeks. Lymphadenopathy is a prominent feature of AOSD seen in about $65 \%$ of patients and must do a biopsy to rule out lymphoma ${ }^{5}$. Life threatening conditions such as hepatic involvement, cardiac tamponade, disseminated intravascular coagulation (DIC), respiratory distress syndrome or pancytopenia were occasionally developed in the course of disease, and some cases were often associated with hemophagocytic syndrome (HS) ${ }^{6}$. Laboratory features of the disease are increased serum levels of C-reactive protein (CRP) and erythrocyte sedimentation rate (ESR), leukocytosis, liver dysfunction, negative results for both rheumatoid factor and antinuclear antibodies, and an increased incidence of hyperferritinemia ${ }^{4}$. Increased serum ferritin level is a nonspecific finding and should not be regarded as a diagnostic test. That ferritin may be helpful for monitoring disease activity during treatment ${ }^{5}$.

Non-steroidal anti-inflammatory drugs (NSAIDs), such as aspirin, ibuprofen or naproxen, help to reduce inflammation ${ }^{5}$. People with high-fever spikes, severe joint symptoms or complications with their internal organs might require glucocorticoids, such as prednisone (0.5$1 \mathrm{mg} / \mathrm{kg} /$ day $)^{5,7}$. Methotrexate has been used successfully in a small series of people to treat adult Still's disease. It may also be used as a "steroid-sparing agent," meaning that if one gives methotrexate, smaller doses of corticosteroids may be sufficient to control disease 5,8 . Some patients are refractory to these conventional therapies. Tumor necrosis factoralpha (TNF) blockers, include infliximab, adalimumab, etanercept, anti-interleukin-1, antiinterleukin-6 agents, and most recently antiCD20-expressing B-cell antibodies are also effective in some cases, but no evidence of respond to other DMARD (gold, penicillamine). Other experimental drugs, including cyclosporine and anakinra, have also been successful in small groups of people ${ }^{2,5,7}$. Even with treatment, it's difficult to predict the course of adult Still's disease. Some people might only experience a single episode, while for others adult Still's disease may develop occasional flair up or a chronic condition. About one-third of people with the disorder may fall into each of the above groups. Even if the patient is symptoms free sometimes they need to continue medications to control inflammation and prevent complications. So, patient needs at least 6 months of treatment ${ }^{5}$.

\section{Conclusion}

AOSD is an important cause of pyrexia of unknown origin (PUO). It needs proper documentation of fever, exclusion of other diseases and observation for at least 6-8 weeks to diagnose the disease. But a definite diagnosis and proper managements can make the prognosis much better.

\section{References}

1. Magadur JG, Billaud E, Barrier JH et al. Epidemiology of adult Still's disease: estimate of the incidence by a retrospective study in west France. Ann Rheum Dis 1995; 54: 587-90.

2. Chakr R, Dora JM, Nogueira FL, Seligman R: Adult-onset still's disease evolving with multiple organ failure: case report and literature review; CLINICS 2007;62(5):645-646.

3. Larson EB: Adult Still's disease-Recognition of a clinical syndrome and recent experience-University of Washington [Specialty Conference]. West J Med, May 1985; 142: 665-671.

4. Otrock Z k, Hatoum HA, Uthman IW, Taher AT, Saab $S$ and Shamseddine AI; Non-Hodgkin's lymphoma in a woman with adult-onset Still's disease: a case report. J of Med Case Reports, 2008; 73(2): $1-4$

5. Ebrahim RA, Oraibi AKA, Mahdi N, Zaber K, Fareed E: Adult Onset Still's Disease (AOSD) - A Case Report; Bahrain Medical Bulletin, September 2002; 24(3): 1-6.

6. Park JH, Bae JH, Choi YS, Lee HS, Jun JB, Jung S, Yoo DH, Bae SC, Kim TH: adult-onset still's disease with disseminated intravascular coagulation and multiple organ dysfunctions dramatically treated with cyclosporine a; J Korean Med Sci 2004; 19: 137-41.

7. Stoica GS, Cohen RI, Rossoff LJ: Adult Still's disease and respiratory failure in a 74 year old woman. Postgrad Med J 2002; 78: 97-98.

8. Nakahara H, Mima T, Hoshino NY, Matsushita M, Hashimoto J, Nishimoto $\mathrm{N}$; A case report of a patient with refractory adult-onset Still's disease who was successfully treated with tocilizumab over 6 years. Japan College of Rheumatology 2008. 